Check for updates

Cite this: RSC Adv., 2017, 7, 49953

Received 1st September 2017 Accepted 13th October 2017

DOI: 10.1039/c7ra09712c

rsc.li/rsc-advances

\section{Self-emulsification of eugenol by modified rice proteins to design nano delivery systems for controlled release of caffeic acid phenethyl ester}

\author{
Tao Wang, ${ }^{\text {abcd }}$ Huaiqiong Chen, ${ }^{d}$ Ren Wang, ${ }^{\text {abc }}$ Zhengxing Chen (D) abc \\ and Qixin Zhong ${ }^{d}$
}

\begin{abstract}
The self-emulsification of oils containing dissolved solutes is not only an intriguing phenomenon but is also of practical interest in the delivery of agricultural chemicals and drugs. Herein, a facile one-step selfemulsification of eugenol with tunable release rates of encapsulated lipophilic compounds was enabled simply by gently mixing eugenol with modified rice proteins (MRPs). Due to binding with eugenol by hydrophobic interactions lowering the oil/water interfacial tension, MRPs underwent secondary structure changes and precipitated on eugenol droplets of nanoscale. Increasing the MRP : eugenol ratio enabled the formation of more porous interfacial films to increase the first-order release rate of eugenol and the resultant anti-proliferation activity of encapsulated caffeic acid phenethyl ester (CAPE) against HTC-116 human colorectal cancer cells. Therefore, novel emulsions with varied interfacial structures can be fabricated utilizing MRPs to control release characteristics and bioactivity of lipophilic compounds.
\end{abstract}

\section{Introduction}

Oil-in-water $(\mathrm{O} / \mathrm{W})$ emulsions are studied for applications such as bio-separation, ${ }^{1}$ drug delivery, ${ }^{2}$ and functional foods. ${ }^{3}$ For practical applications, emulsions shall be prepared with practical technologies, and droplets must be kinetically stable against destabilization mechanisms of creaming, aggregation, coalescence, and Ostwald ripening during shelf-life storage. ${ }^{4}$ Similar to conventional methods of fabricating micro- or nanoscale structures, emulsions can be prepared with top-down or bottom-up approaches. ${ }^{5}$ Top-down approaches require intensive mechanical energy in unit operations such as high shear and high pressure homogenization, microfluidization, colloidal mills, and ultrasonication. In contrast, bottom-up approaches generally do not require high mechanical energy but are driven by physical, chemical, and biological means. Each approach has pros and cons and may find unique applications.

Self-emulsification refers to the formation of emulsions according to thermodynamics requiring little mechanical energy. ${ }^{6}$ One common approach is to dissolve overall water-

${ }^{a}$ State Key Laboratory of Food Science and Technology, Jiangnan University, Wuxi 214122, People's Republic of China. E-mail: zxchen_2008@126.com; Fax: +86 510 85197856; Tel: +8651085197025

${ }^{b}$ National Engineering Laboratory for Cereal Fermentation Technology, Jiangnan University, Wuxi 214122, People's Republic of China

'School of Food Science and Technology, Jiangnan University, Wuxi 214122, People's Republic of China

${ }^{d}$ Department of Food Science and Technology, University of Tennessee, Knoxville, TN, 37996-4539, USA. E-mail: qzhong@utk.edu; Fax: +1 865974 7332; Tel: +1 865974 6196 soluble surfactants in an oil that was then mixed with an aqueous phase, which results in the diffusion of surfactants to the aqueous phase and the simultaneous inclusion of oil as droplets. ${ }^{7}$ This approach is commonly studied for synthetic surfactants that can be tailored for hydrophile-lipophile balance properties but may have toxicity concerns. ${ }^{8}$ Another group of methods is to control the solubility of oils. For example, eugenol, with $\mathrm{p} K_{\mathrm{a}}$ of 10.19 at $25{ }^{\circ} \mathrm{C},{ }^{9}$ can be first deprotonated at alkaline $\mathrm{pH}$ to make it soluble before readjusting to neutral acidity to induce protonation and therefore phase separate into droplets that can be in situ adsorbed with surfactants in the aqueous phase. ${ }^{10}$ This approach can be studied for many water-soluble biopolymeric surfactants such as proteins and polysaccharides, but the extreme $\mathrm{pH}$ used to dissolve oil phase compounds can cause the degradation of many bioactive compounds, such as cleavage of the ester bond of caffeic acid phenethyl ester (CAPE) that has shown excellent activities against the proliferation of various cancer cells. ${ }^{11}$ Another approach is to control the interactions among proteins by first dissolving proteins in the continuous phase followed by altering polarity or acidity to cause anti-solvent precipitation of proteins on oil-water interface to form $\mathrm{O} / \mathrm{W}$ emulsions. For example, zein is a group of corn proteins soluble at $55-90 \%$ aqueous ethanol, and the slow addition of water into a mixture of soya oil, zein, and $60 \%$ aqueous ethanol resulted in precipitation of zein on oil droplets with a dimension of approximately $30-40 \mu \mathrm{m} .{ }^{12}$ In our recent study using modified rice proteins (MRPs) that are soluble above $\mathrm{pH} 7.0$ but drastically lose the solubility from $\mathrm{pH} 7.0$ to 6.0 , titration of mixtures with soya oil and basic MRPs solution at $\mathrm{pH}$ 9.0 to $\mathrm{pH}$ 6.2-7.0 induced the precipitation of MPRs on oil 
droplets with the $\mathrm{pH}$-dependent thickness, which subsequently controlled the release rate of $\beta$-carotene encapsulated in emulsion droplets during simulated in vitro digestions. ${ }^{13}$ However, the need to slowly control solvent chemistry to manipulate protein solubility makes it difficult to scale up these systems.

Herein, we report a straightforward emulsifying protocol based on guest-host reaction between eugenol and modified without high mechanical energy or surfactant-assistance. Because the deprotonation of eugenol can lower $\mathrm{pH}$ to change the solubility of MRPs, simply mixing eugenol and MRPs at slightly alkaline $\mathrm{pH}$ of 8.0 can induce the precipitation of MRPs. Due to hydrophobic binding between MRPs and eugenol, the deposition is steered to the surface of oil droplets to selfemulsify eugenol. The surfactant-free emulsions are stable in droplet sizes during 4 week storage. We further demonstrate that the emulsion droplets produced from different ratios of MRP : eugenol have different structures to enable the controlling of release rate of emulsion droplet components that can be used to control biological activities of encapsulated CAPE.

\section{Results and discussion}

\section{Properties of emulsions}

The green, facile, and spontaneous emulsification was enabled by gently mixing eugenol with MRPs solutions (600 rpm) at room temperature $\left(\mathrm{RT}, \sim 21{ }^{\circ} \mathrm{C}\right), \mathrm{pH} 8.0$ for $1 \mathrm{~h}$. Table 1 shows the efficiency of encapsulating (EE) and percentages of MRPs and eugenol in fresh emulsions. As the MRP : eugenol ratio increased, the EE increased rapidly because more MRPs were available to emulsify the lowered amount of eugenol, and the $\mathrm{MRP} \%$ in emulsions increased, which indicates the enhanced retention of MRPs after forming emulsions. Because eugenol has a water solubility of $2.4 \mathrm{mg} \mathrm{mL}{ }^{-1}(0.23 \% \mathrm{v} / \mathrm{v})$ at $25{ }^{\circ} \mathrm{C},{ }^{14}$ results in Table 1 confirmed the successful formation of emulsions. Fig. 1 shows the atomic force microscopy (AFM) images of MRPs and emulsion droplets. MRPs after drying had a dimension of $c a$. 20-40 nm (Fig. 1a). Upon mixing with eugenol, defined spherical particles with a diameter of 80-250 $\mathrm{nm}$ were observed for emulsions prepared with a MRP : eugenol ratio from $1: 1$ to $1: 20$ (Fig. 1b-e). As the MRP: eugenol ratio reached $1: 30$, irregular aggregates were observed (Fig. 1f).
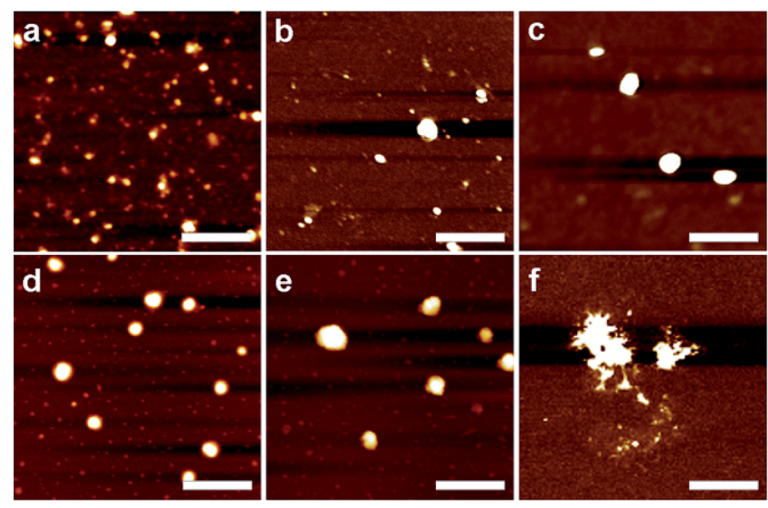

Fig. 1 Topographical AFM images of MRP (a) and eugenol droplets prepared at a MRP : eugenol (g/mL) ratio of $1: 1$ (b), $1: 4$ (c), $1: 10$ (d), $1: 20$ (e) and $1: 30$ (f). Scale bar $=400 \mathrm{~nm}$.

Dynamic light scattering (DLS) results (Fig. 2a) generally agreed with particle dimensions in AFM (Fig. 1). There were two peaks observed for MRPs, with the smaller major peak centering on $20 \mathrm{~nm}$ and the larger on $250 \mathrm{~nm}$. The smaller peak shifted to the larger size regime and became wider as the MRP : eugenol ratio decreased, eventually evolving to a peak centered on $\sim 2000 \mathrm{~nm}$ at a MRP : eugenol ratio of $1: 30$. The AFM and DLS results further confirmed the formation of emulsions at the studied conditions.

The emulsion prepared at a MRP : eugenol ratio of $1: 30$ precipitated after $24 \mathrm{~h}$ storage at RT and was not studied further, whereas other samples prepared at larger MRP : eugenol ratios from $1: 20$ to $1: 0.5$ did not show macroscopic phase separation after 4 week storage at RT. The treatment with a MRP : eugenol ratio bigger than 1:4 was also not studied further because of a relatively low oil loading (Table 1). Changes of mean hydrodynamic diameters and distributions of emulsions prepared with MRP : eugenol ratios of $1: 4,1: 10$, and $1: 20$ during 4 week storage at RT are presented in Fig. 2 . When compared to fresh emulsions (Fig. 2a), the major peak was mostly unchanged after 4 weeks, while the shifting of the secondary peak to larger dimensions was observed. Because these emulsions had a zeta-potential magnitude of above $25 \mathrm{mV}$ (Table 1) that typically is strong enough to prevent the

Table 1 Efficiency of encapsulating (EE) eugenol and MRP and eugenol concentrations in emulsions prepared with an aqueous phase with $1 \%$ w/ $\checkmark$ MRPs and various MRP : eugenol ratios ${ }^{a}$

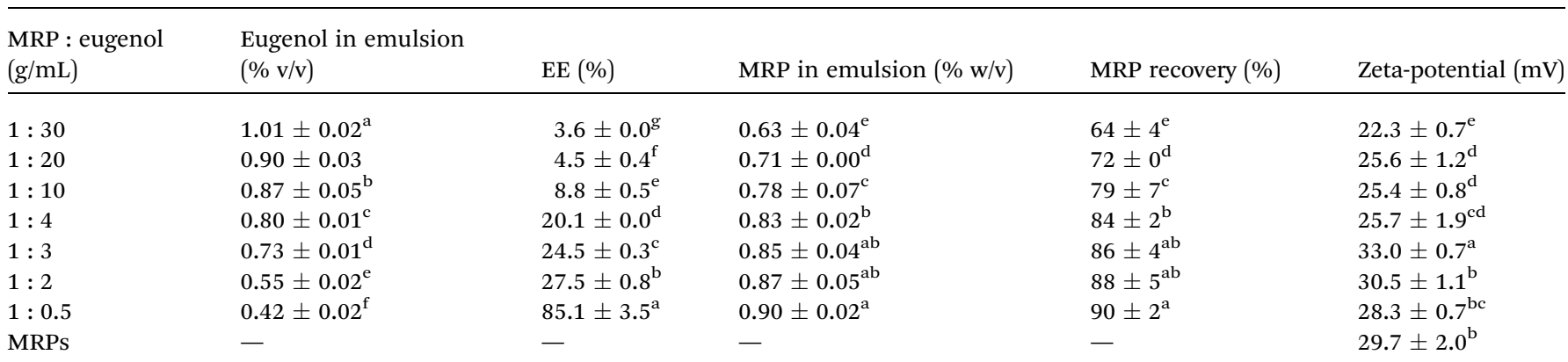

${ }^{a}$ Numbers are mean $\pm \mathrm{SD}(n=3)$. Different superscript letters in the same column indicate significant difference in the mean $(p<0.05)$. 

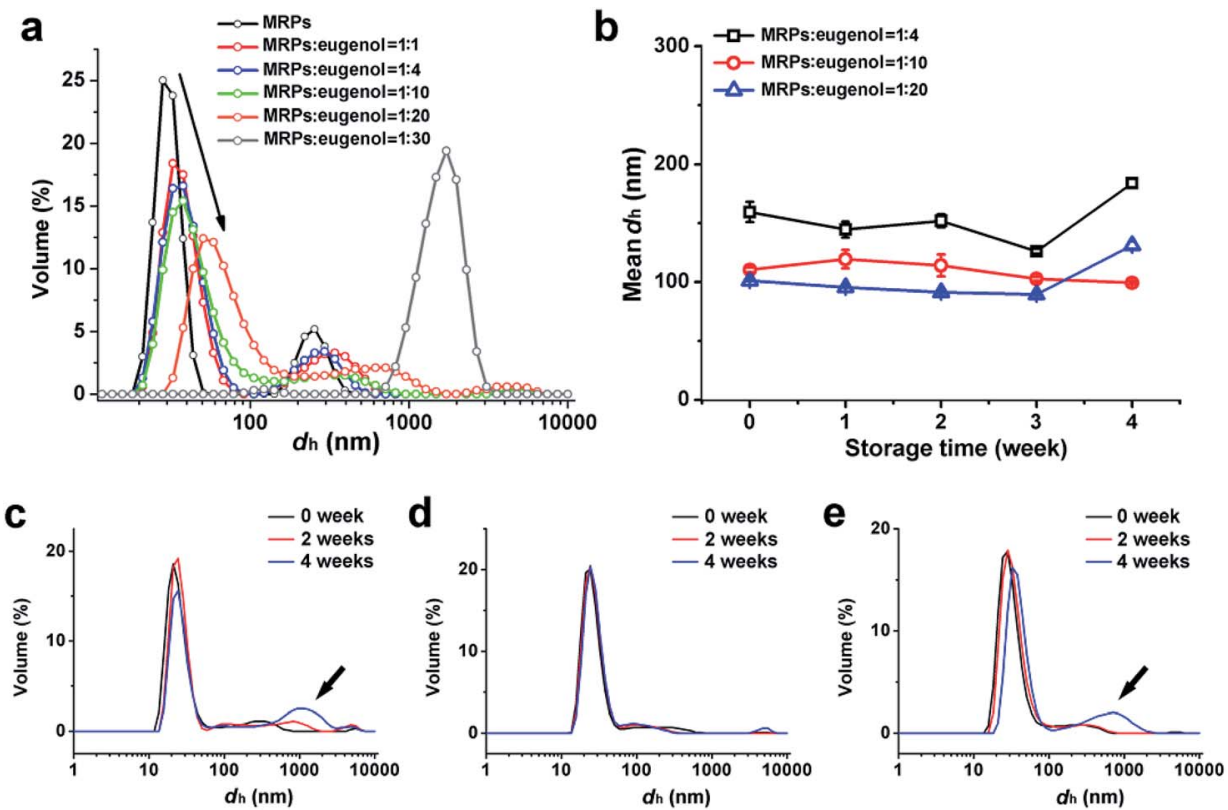

Fig. 2 DLS size distributions of MRPs at pH 8.0 and fresh emulsions prepared at various MRP : eugenol (g/mL) ratios (a), and changes of mean hydrodynamic diameter (dh) (b) and distribution of emulsion droplets prepared at MRP : eugenol ratios of $1: 4$ (c), $1: 10$ (d), and $1: 20$ (e) during storage at RT for up to 4 weeks.

aggregation of oil droplets, ${ }^{15}$ changes in droplet size distributions of emulsions after storage imply the occurrence of Ostwald ripening. ${ }^{16}$ The difference in particle size distribution changes of these three emulsions appeared to have resulted from the interfacial structures, as evidenced in scanning transmission electron microscopy (STEM) and focused ion beam scanning electron microscopy (FIB-SEM) in Fig. 3 that showed the least defined structures of the emulsion prepared with a MRP : eugenol ratio of $1: 20$ corresponding to the most significant increase of droplet size at week 4 (Fig. 2e).

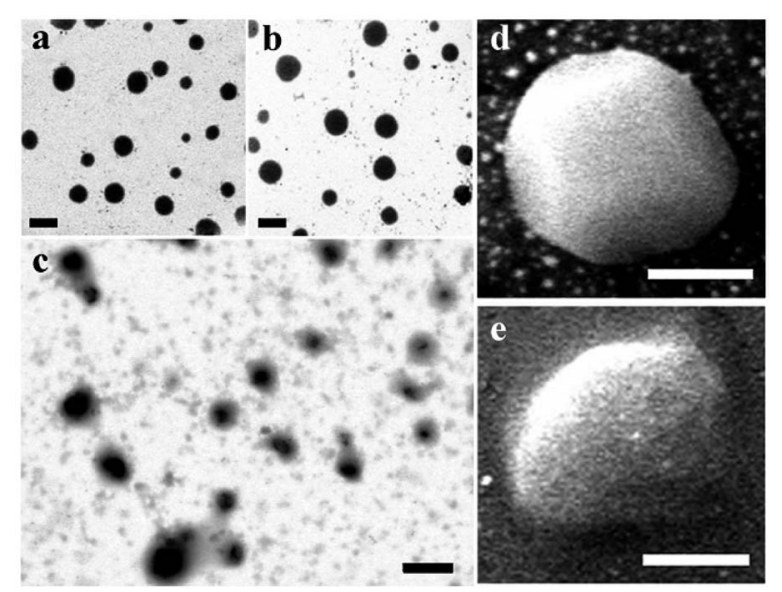

Fig. 3 Droplet structures studied using STEM for eugenol emulsions prepared at a MRP : eugenol $(\mathrm{g} / \mathrm{mL})$ ratio of $1: 4(\mathrm{a}), 1: 10(\mathrm{~b})$, and $1: 20$ (c), respectively, or FIB-SEM for emulsions prepared at a MRP : eugenol ratio of $1: 4$ (d) and $1: 20$ (e). Scale bars $=200 \mathrm{~nm}$ in (a), (b), (c) or $100 \mathrm{~nm}$ in (d) and (e).

\section{Physical mechanisms of self-emulsification}

Emulsion $\mathbf{p H}$. Eugenol can be deprotonated, with $\mathrm{p} K_{\mathrm{a}}$ of 10.19 at $25^{\circ} \mathrm{C},{ }^{8}$ which was verified by the $\mathrm{pH}$ of water-eugenol mixture (Fig. 4). According to the Henderson-Hasselbalch equation, ${ }^{17}$ the $\mathrm{pH}$ of saturated eugenol-water mixtures is 6.0 that was close to the measured pH 5.9 (Fig. 4). In contrast, all emulsions had a $\mathrm{pH}$ higher than 7.8, and the reduction of $\mathrm{pH}$ was not significantly different $(p>0.05)$. The smaller $\mathrm{pH}$ reduction of emulsions than water-eugenol mixtures is likely due to the buffering capacity of MRPs with acidic amino acids binding with protons. As presented previously, the reduction of $\mathrm{pH}$ to be below 7.0 enabled the precipitation of MRPs on soya oil

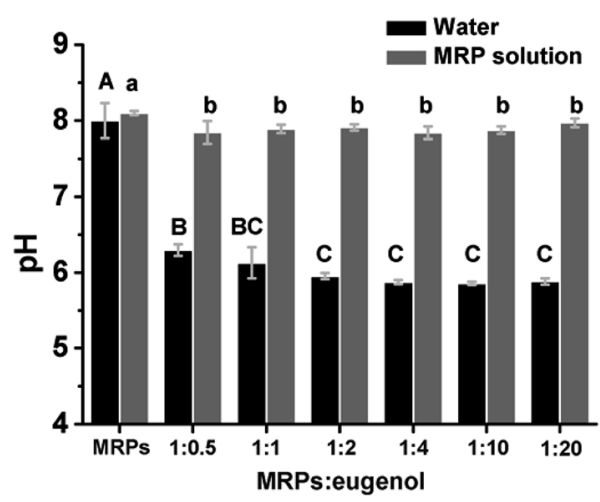

Fig. $4 \mathrm{pH}$ of emulsions prepared with $1 \%$ w/v MRP solution at $\mathrm{pH} 8.0$ and different MRP : eugenol ratios, in comparison to simple mixtures with water adjusted to 8.0 and same amounts of eugenol. Error bars are SD $(n=3)$. Different lower- or uppercase letters above bars indicate significant differences within the same group $(p<0.05)$. 

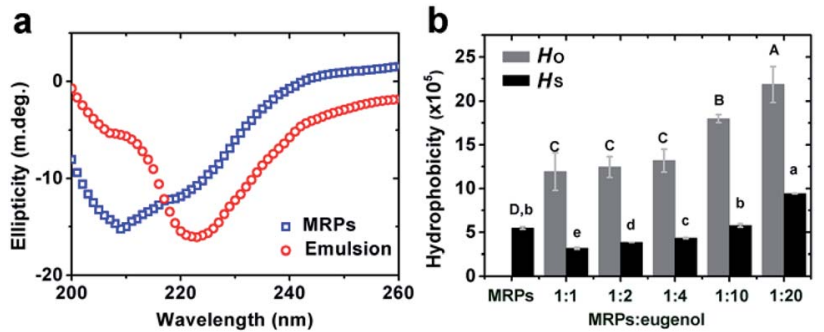

Fig. 5 Comparison of far-UV CD spectra of MRPs before and after emulsification at a MRP : eugenol ratio of $1: 1$ (a), and the overall hydrophobicity $\left(H_{O}\right)$ and surface hydrophobicity $\left(H_{S}\right)$ of MRPs estimated for emulsions prepared with various MRP : eugenol ratios (b). Error bars are SD $(n=3)$. Different lower- or uppercase letters above bars indicate significant differences within the same group $(p<0.05)$.

droplets, ${ }^{13}$ which likely was the case in the present study at the eugenol-water interface. However, no acidification is needed in the present study, and the emulsions maintained the acidity enabling the solubility of MRPs, making it a novel selfemulsification technology.

Structural changes of MRPs. Structural changes of MRPs after emulsification were first studied with far-UV CD spectra using the emulsion prepared with a MRP : eugenol ratio of $1: 1$. After emulsification, the negative ellipticity maxima showed the decrease and increase of intensities around $210 \mathrm{~nm}$ and $220 \mathrm{~nm}$ (Fig. 5a), respectively, indicating a helix-sheet transition ${ }^{\mathbf{1 8}}$ that is evident by changes in percentages of secondary structures (Table 2). $\alpha$-Helix structures play a key role in stabilization of proteins by maximizing polar-polar interactions and minimizing aploar-polar contacts, ${ }^{\mathbf{1 9}}$ whereas $\beta$-sheet structures are intermediates of protein aggregation due to hydrophobic interactions. ${ }^{20}$ Therefore, changes in secondary structures of MRPs enable the deposition of MRPs on eugenol during emulsification. The impacts of emulsification on MRP structures were further studied for $H_{\mathrm{O}}$ and $H_{\mathrm{S}}$ based on fluorescence of ANS. The $H_{\mathrm{O}}$ was increased by addition of a greater amount of eugenol (Fig. 5b). This agrees with the reduction of MRP\% in emulsions (Table 1) that is expected to be due to the exposure of hydrophobic amino acid residues after adsorbing onto eugenol droplets. The relatively low $H_{\mathrm{S}}$ (Fig. 5b) on the other hand agreed with considerable surface charges of the emulsions (Table 1) and the overall solubility of MRPs at pH above 7.8 (Fig. 4).

Reduction of interfacial tension. As presented in Fig. 6, the force needed to pull the eugenol-water interface and therefore $\gamma$ was significantly reduced by MRPs dissolved in water. In addition, the breakdown of the $\mathrm{O} / \mathrm{W}$ interface was well ahead for the

Table 2 Percentages of secondary structures of MRPs and emulsion prepared with a MRPs/eugenol ratio of $1: 1^{a}$

\begin{tabular}{llrll}
\hline & $\alpha$-Helix & \multicolumn{1}{c}{$\beta$-Sheet } & \multicolumn{1}{l}{$\beta$-Turn } & Random coil \\
\hline MRPs & $40.7 \pm 1.7$ & $7.5 \pm 0.4$ & $33.3 \pm 1.2$ & $18.5 \pm 0.1$ \\
Emulsion & $23.4 \pm 2.0$ & $24.8 \pm 1.1$ & $36.1 \pm 2.7$ & $15.7 \pm 0.6$
\end{tabular}

${ }^{a}$ Values represent mean $\pm \mathrm{SD}(n=3)$.
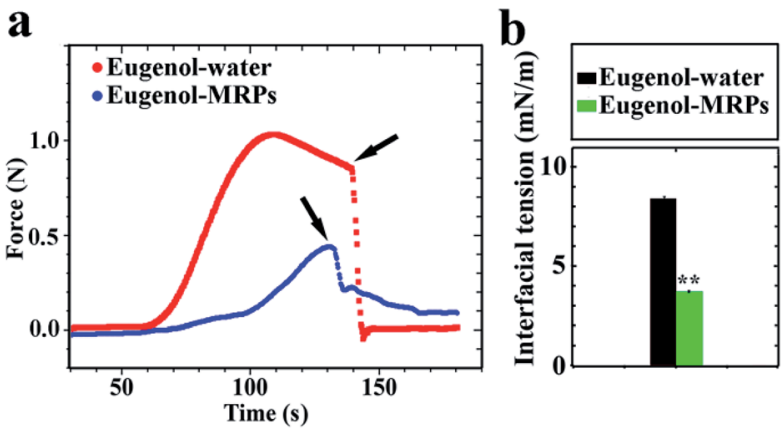

Fig. 6 Force required to pull a Du Noüy ring from a bottom eugenol phase to an upper aqueous phase with water adjusted to $\mathrm{pH} 8.0$ or a $1 \% \mathrm{w} / \mathrm{v}$ MRP aqueous solution at $\mathrm{pH} 8.0$ (a), and the interfacial tension estimated from the maximum pulling force (b). Arrows show the breakup of $\mathrm{O} / \mathrm{W}$ interface during pulling the Du Noüy ring. Error bars are SD $(n=3)$. The notation "**" indicates a significant difference between the two treatments $(p<0.01)$.

aqueous phase with MRPs, indicating the facilitated formation of interfacial films by MRPs. ${ }^{21}$ The $\gamma$ of the eugenol-water interface at $25{ }^{\circ} \mathrm{C}$ was around $8.5 \mathrm{mN} \mathrm{m}^{-1}$, close to the value of $9.0 \mathrm{mN} \mathrm{m}^{-1}$ measured based on contact angles of eugenol droplets on flat glass substrates immersed in water ${ }^{22}$ and $c a .7$ $\mathrm{mN} \mathrm{m}{ }^{-1}$ measured with the pendant-drop method. ${ }^{23}$ The $\gamma$ was significantly ( $p<0.01$ ) lowered to $3.5 \mathrm{mN} \mathrm{m}^{-1}$ at the presence of $1 \% \mathrm{w} / \mathrm{v}$ MRPs in the aqueous phase. The results suggested that the marked decrease in $\mathrm{O} / \mathrm{W}$ interfacial tension was responsible for the formation of nano-scale eugenol droplets after selfemulsification (Fig. 2).

\section{Molecular binding between MRPs and eugenol}

Fluorescence spectroscopy. The emission spectra of emulsions prepared with ANS are shown in Fig. 7a. A greater amount of eugenol used to prepare emulsions corresponded to a significant increase in the characteristic fluorescence intensity of ANS centered at $450 \mathrm{~nm}$, indicating the enhanced molecular

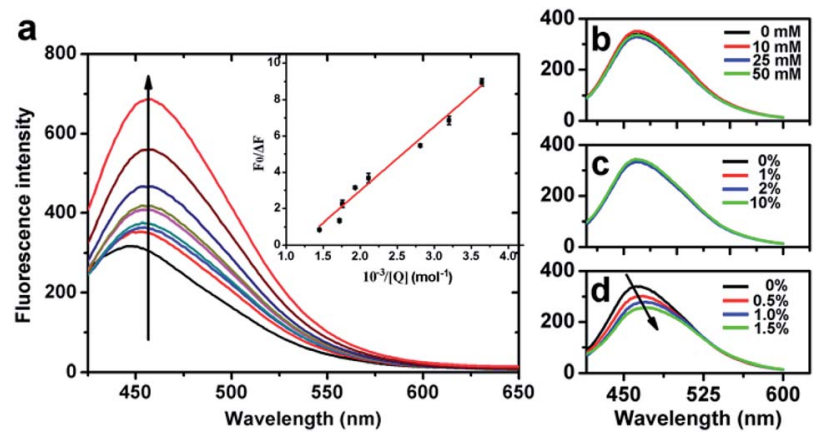

Fig. 7 Fluorescence spectra at $298 \mathrm{~K}$ of ANS-containing fresh emulsions prepared with MRP : eugenol ratios of $1: 0,1: 0.5,1: 1,1: 2$, $1: 3,1: 4,1: 10,1: 20$ and $1: 30$ (upward direction following the arrow) after 100-time dilution with distilled water (a), as well as emulsions prepared with a MRP : eugenol $(\mathrm{g} / \mathrm{mL})$ ratio of $1: 10$ and supplemented with different concentrations of urea (b), $\mathrm{NaCl}$ (c), and SDS (d) in the aqueous phase before emulsification. 
Table 3 Stern-Volmer binding constant determined from fluorescence spectroscopy and enthalpy $(\Delta H)$, entropy $(\Delta S)$, and Gibbs free energy $(\Delta G)$ changes and binding stoichiometry $(n)$ of eugenol with MRPs determined in ITC $^{a}$

\begin{tabular}{|c|c|c|c|c|}
\hline$K_{\mathrm{a}}\left(10^{3} \mathrm{M}^{-1}\right)$ & $\Delta H\left(\mathrm{~kJ} \mathrm{~mol}^{-1}\right)$ & $\Delta S\left(\mathrm{~J} \mathrm{~mol}^{-1}\right)$ & $\Delta G\left(\mathrm{~kJ} \mathrm{~mol}^{-1}\right)$ & $n$ \\
\hline $1.17 \pm 0.07$ & $5.09 \pm 0.04$ & $37.25 \pm 1.63$ & $-6.01 \pm 0.44$ & $10 \pm 2.1$ \\
\hline
\end{tabular}

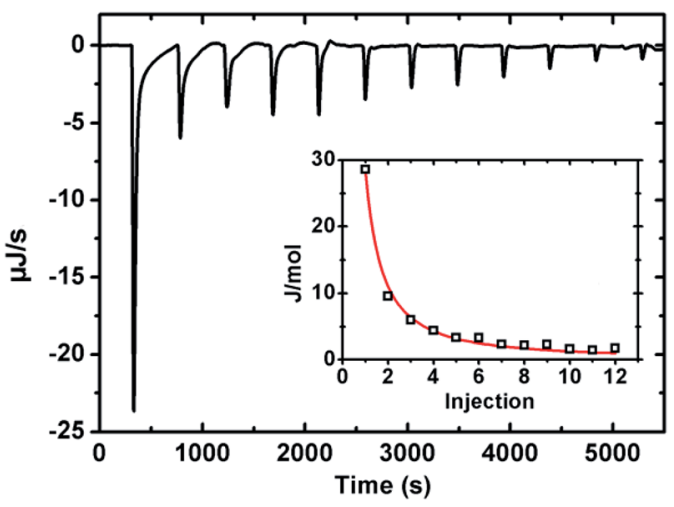

Fig. 8 Thermogram at $298 \mathrm{~K}$ when titrating $500 \mu \mathrm{L}$ of $0.5 \%$ MRP stock solutions with eugenol at $2.5 \mu \mathrm{L}$ per injection. The inset curve shows the fitting of data to a one-set model with the algorithm of the instrument.

binding between eugenol and MRPs. ${ }^{24}$ The peak fluorescence intensity (inset of Fig. 7a) was fit to a modified Stern-Volmer equation (eqn (1)) to estimate the associative binding constant of the two molecules, which was determined to be $1.17 \times 10^{3}$ $\mathbf{M}^{-1}$ (Table 3) that indicates the complexation of two molecules. ${ }^{25}$

$$
\frac{F_{0}}{\Delta F}=\frac{F_{0}}{F-F_{0}}=\frac{1}{f_{\mathrm{a}} K_{\mathrm{a}}} \frac{1}{[\mathrm{Q}]} \pm \frac{1}{f_{\mathrm{a}}}
$$

where $F_{0}$ and $F$ are the fluorescence intensity in the absence and presence of eugenol, respectively, $f_{\mathrm{a}}$ is the fraction of accessible fluorescence, $Q$ is the molar concentration of eugenol, and $K_{\mathrm{a}}$ is an associative binding constant between a ligand and an acceptor.

Physical forces enabling molecular binding between proteins and small molecular ligands can be further examined by mixing with reagents of urea, sodium dodecyl sulfate (SDS), and ions that weaken hydrogen bonding, hydrophobic interactions, and electrostatic interactions, respectively. ${ }^{26}$ The significant reduction of fluorescence intensity by SDS (Fig. 7d) but not urea (Fig. 7b) or $\mathrm{NaCl}$ (Fig. 7c) suggests the major contribution of hydrophobic interactions to the binding between MRPs and eugenol.

Isothermal titration calerimetry (ITC). Fig. 8 shows a representative thermograph during the titration of eugenol into the MRP stock solution at $298 \mathrm{~K}$. The negative $\Delta G$ indicates the endothermic event was thermodynamically favored, and the positive $\Delta H$ and $\Delta S$ suggest the process is entropy-driven (Table 3 ). Therefore, both fluorescence spectroscopy and ITC results support the molecular and thermodynamic bases of selfemulsification of eugenol by MRPs.

\section{Properties of emulsions as a potential delivery system of CAPE}

The properties of emulsions as a potential delivery system were first characterized for release characteristics of eugenol to understand interfacial structures as affected by emulsion preparation conditions. CAPE was not studied for release kinetics because of its low water solubility. The release profiles of eugenol in Fig. 9a are characteristics of the first-order release kinetics, ${ }^{27}$ and the following model can be used to estimate kinetic parameters: ${ }^{28}$

$$
Q \%=1-\mathrm{e}^{-k t}
$$

where $Q \%$ is the percentage of eugenol released at time $t$, and $k$ is the release rate constant. For all three emulsions, the $R^{2}$ values after fitting the data to the model (Fig. 9b) were greater than 0.978 (Table 4), confirming the first-order release kinetics. The $k$ value increased with the decreasing MRP : eugenol ratio, which suggests the more porous interfacial film at a higher content of eugenol, as previously presented for microscopy results (Fig. 3). Therefore, these emulsions are expected to have
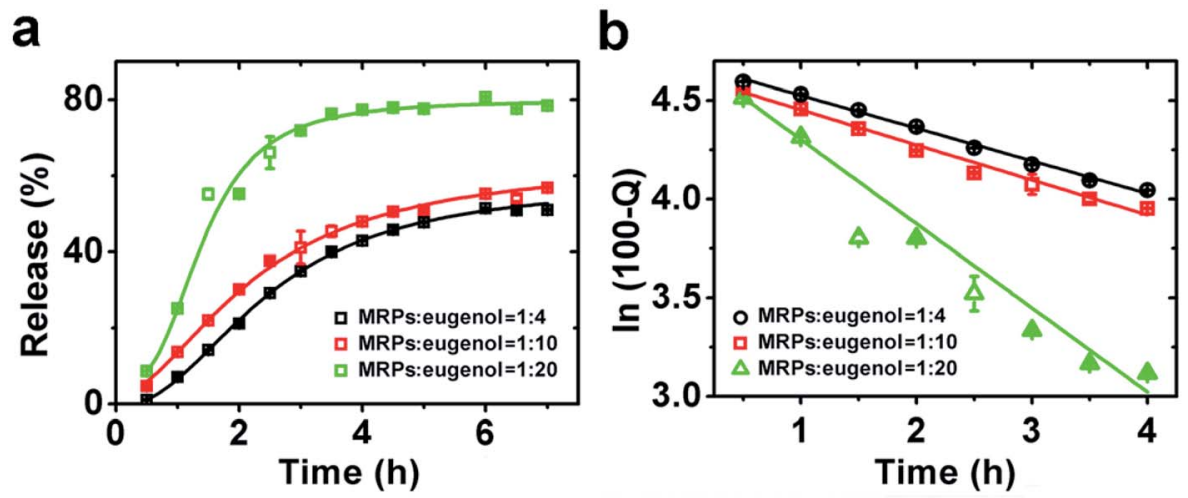

Fig. 9 Release kinetics of eugenol from emulsions prepared at MRP : eugenol ( $\mathrm{g} / \mathrm{mL}$ ) ratios of $1: 4,1: 10$ and $1: 20$ (a), and the fitting of data according to the first order release kinetics (b). 
Table $4 R^{2}$ of fitting eugenol release data to the first order kinetics model and the estimated release rate constant $(k)$ from emulsions prepared with three MRP : eugenol ratios

\begin{tabular}{lll}
\hline $\begin{array}{l}\text { MRPs : eugenol } \\
(\mathrm{g} / \mathrm{mL})\end{array}$ & $R^{2}$ & $k^{a}$ \\
\hline $1: 4$ & 0.995 & $0.165 \pm 0.001^{\mathrm{b}}$ \\
$1: 10$ & 0.985 & $0.180 \pm 0.003^{\mathrm{b}}$ \\
$1: 20$ & 0.978 & $0.409 \pm 0.002^{\mathrm{a}}$
\end{tabular}

${ }^{a}$ Values are mean $\pm \mathrm{SD}(n=3)$. Different superscript letters indicate significant difference $(p<0.05)$.

different release kinetics of encapsulated drugs. The three emulsions were then prepared with CAPE and studied for antiproliferation activity against HCT-116 cells, in comparison to same amounts of CAPE dissolved in DMSO. The control emulsions without CAPE did not show noticeable impacts on the cytotoxicity (data not shown), indicating the negligible activity of eugenol at the studied conditions. Overall, the cell viability tested at a CAPE concentration of $20 \mu \mathrm{g} \mathrm{mL} \mathrm{m}^{-1}$ was lower than a dose of $2.0 \mu \mathrm{g} \mathrm{mL}{ }^{-1}$, and emulsions prepared with different MRP : eugenol ratios showed significantly different antiproliferation activities $(p<0.05)$ (Fig. 10). A higher release rate of eugenol from emulsions (Table 4) seemed to agree with a higher activity of CAPE (Fig. 10) that signifies the possibility of controlling interfacial structures of emulsions to design delivery systems with desirable properties. At both doses, the emulsion prepared with a MRP : eugenol ratio of 1:20 resulted in a significantly $(p<0.05)$ lower cell viability than the same amount of CAPE dissolved in DMSO. Therefore, delivery systems based on the studied emulsion can be used to not only disperse CAPE but also enhance the activity. Previously, such enhancement of anti-proliferation activity was reported for delivery systems of curcumin nanoencapsulated in sodium caseinate, ${ }^{29}$ curcumin encapsulated in silk fibroin nanofibrous matrix, ${ }^{30}$ and doxorubicin loaded in amphiphilic peptide

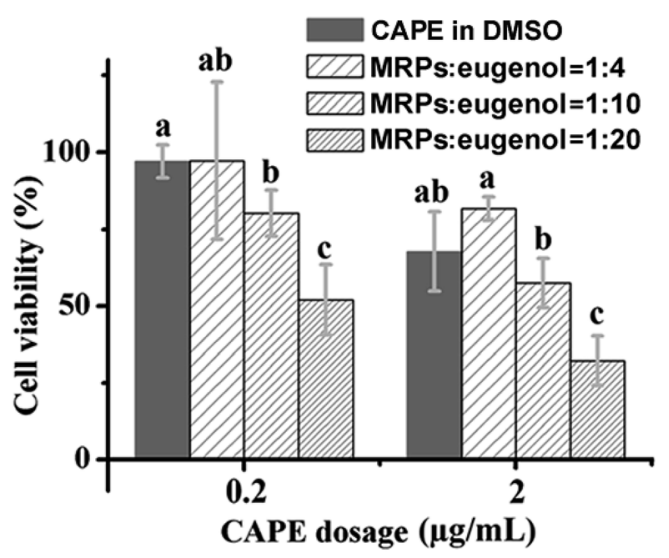

Fig. 10 Normalized cell viability after treatment by 0.2 or $2.0 \mu \mathrm{g} \mathrm{mL}^{-1}$ CAPE dissolved in DMSO or loaded in emulsions prepared at different MRP : eugenol $(\mathrm{g} / \mathrm{mL})$ ratios. Error bars are SD $(n=4)$. Different lowercase letters above bars indicate significant differences within the same dose group $(p<0.05)$. dendritic copolymer nano-conjugates. ${ }^{31}$ The enhancement was correlated with improved membrane uptake of drug carriers and the accelerated release of drugs was proposed to result from the increased membrane fluidity before drug insertion. ${ }^{32}$

\section{Conclusions}

Findings from the present study showed the complexation of eugenol and MRPs due to hydrophobic interactions. The affinity of the two molecules facilitated the significant reduction of eugenol/water interfacial tension to enable self-emulsification by MRPs precipitated on droplet surface due to local deportation of eugenol lowering $\mathrm{pH}$ to reduce protein solubility. The reduction of interfacial tension to $3.5 \mathrm{mN} \mathrm{m}^{-1}$ enabled the formation of eugenol droplets with a dimension of $80-250 \mathrm{~nm}$ under mild stirring. Fabrication of interfacial structures was enabled by controlling the MRP : eugenol ratio during selfemulsification, which resulted in the controlled release rate of eugenol and the enhanced activity of encapsulated CAPE. The self-assembled emulsions in the present study may provide a novel system to deliver lipophilic bioactive compounds with controllable release properties and bioactivities.

\section{Experimental section}

\section{Materials}

Rice protein isolate powder containing $90.14 \mathrm{wt} \%$ protein as determined by the Kjeldahl method ${ }^{33}$ was procured from Jinnong Biotechnology Ltd. (Yichun, Jiangxi, China). Eugenol, 8anilino-1-naphthalenes (ANS), CAPE, dimethyl sulfoxide (DMSO) with a purity $>99 \%$, and fetal bovine serum (FBS) were purchased from Sigma-Aldrich Corp. (St. Louis, MO, USA). Human colon cancer cell line HCT-116 was purchased from the American Type Culture Collection (Manassas, VA, USA) and was cultured in McCoy's medium (Mediatech, Herdon, VA, USA). Other chemicals were of an analytical grade and were used without further purification.

\section{Modification of rice proteins}

The preparation of MRPs was described in our previous study. This was done by incubating a mixture with rice protein powder in $0.03 \mathrm{M} \mathrm{NaOH}(1: 30, \mathrm{w} / \mathrm{v})$ overnight at $-20{ }^{\circ} \mathrm{C}$, followed by milling (model XFB-500, Zhongcheng Mechanical Co., Changsha, China). The obtained mixture was brought to room temperature ( $\mathrm{RT}, \sim 21^{\circ} \mathrm{C}$ ) and adjusted to $\mathrm{pH}$ 7.0. After centrifugation at $7000 \mathrm{~g}$ for $10 \mathrm{~min}$, the supernatant was dialyzed against distilled water using a regenerated cellulose membrane (Thermo Scientific Co., Marietta, OH, USA) with a molecularweight-cut-off of $1000 \mathrm{Da}$. The desalted protein solution was lyophilized, and the obtained powder was referred as the MRPs in following experiments.

\section{Preparation of emulsions}

The aqueous phase was prepared by hydrating $1.00 \mathrm{~g}$ MRPs in $100.0 \mathrm{~mL}$ distilled water under stirring at RT for $1 \mathrm{~h}$ and adjusting $\mathrm{pH}$ to 8.0 using $0.1 \mathrm{M} \mathrm{NaOH}$ as the MRP stock 
solution. As reported previously, MRPs were well-dissolved at pH 8.0 but quickly lose solubility when $\mathrm{pH}$ was under 7.0. ${ }^{13}$ Eugenol was mixed with the MRP stock solution at various MRP : eugenol $\left(\mathrm{g} \mathrm{mL}^{-1}\right)$ ratios on a stirring plate (model Poly 15, Thermo Scientific Co., Marietta, OH, USA) at a speed of $600 \mathrm{rpm}$ and RT for $1 \mathrm{~h}$. The mixtures were then centrifuged at $6000 \mathrm{~g}$ for $10 \mathrm{~min}$ using a Sorvall RC 5B PLUS centrifuge (Thermo Scientific Co., Marietta, OH, USA). The serum phase was obtained as the emulsion for characterization. Control MRP solutions were prepared without eugenol, following identical procedures.

\section{Properties of emulsions}

pH. The $\mathrm{pH}$ of fresh emulsions was measured with a model $51302910 \mathrm{pH}$ meter (Mettler-Toledo International Inc., Columbus, OH, USA) at RT. The $\mathrm{pH}$ of mixtures with same amounts of distilled water and eugenol after being processed at above conditions was also measured for comparison to the corresponding emulsions.

Composition of emulsions. A fresh emulsion was diluted by 10 times $(\mathrm{v} / \mathrm{v})$ with ethanol that is a good solvent of eugenol but a poor solvent of MRPs. After stirring overnight at RT in a dark place, the mixture was centrifuged at $6000 \mathrm{~g}$ for $10 \mathrm{~min}$ using the above centrifuge. The supernatant was transferred and diluted using ethanol to measure the absorbance at $287 \mathrm{~nm}$ (Evolution 201, Thermo Scientific Co., Marietta, OH, USA), which was used to determine the amount of eugenol based on a standard curve prepared with standard solutions with various amounts of eugenol dissolved in ethanol. The precipitate was flushed with water and amounts of proteins were determined by the Kjeldahl method. ${ }^{33}$ Encapsulation efficiency (EE) was calculated as the percentage of eugenol mass in an emulsion with respect to the total eugenol mass used in emulsion preparation.

Size distribution and zeta-potential. The size distribution and zeta-potential of droplets were determined at RT using a Malvern Nano ZS instrument (Malvern Instrument Ltd, Worcestershire, UK). All samples were diluted with distilled water for 100 times and tested at least in triplicate.

Atomic force microscopy (AFM). MRP solutions and fresh emulsions were diluted by 250 times with distilled water. $5 \mu \mathrm{L}$ of a diluted sample was spread on a freshly-cleaved mica sheet with an area of approximate $1.8 \mathrm{~cm}^{2}$ and dried at RT in a dark cabinet overnight $(\sim 12 \mathrm{~h})$. Topographical images were collected at the tapping mode using a nano-probe cantilever tip (Bruker Nanoprobe, Camarillo, CA, USA) at a frequency from 50 to 100 $\mathrm{kHz}$ on a Multimode VIII microscope (Bruker Corporation, Billerica, MA, USA). Images were analyzed using the AFM instrument software (Nanoscope Analysis version 1.50, Bruker Corporation, Billerica, MA, USA).

Scanning transmission electron microscopy (STEM). $10 \mu \mathrm{L}$ of a fresh emulsion was casted on a holey carbon grid that was then kept flat for $1 \mathrm{~min}$ to allow sample attachment. The sample was then tapped briefly with a filter paper to remove residual liquid, and STEM images were obtained using a Zeiss Auriga 60 dual beam microscope (Carl Zeiss Microscopy, Oberkochen, Germany) operated at an accelerating voltage of $30 \mathrm{keV}$.
Focused ion beam scanning electron microscopy (FIB-SEM). $10 \mu \mathrm{L}$ of a fresh emulsion was casted on a double-sided conductive tape mounted on a sample holder and was dried overnight $(\sim 12 \mathrm{~h})$ at RT. A Zeiss Auriga 60 FIB-SEM instrument (Carl Zeiss Microscopy, Oberkochen, Germany) equipped with a FIB probe operating at a voltage of $30 \mathrm{kV}$ and $50 \mathrm{pA}$ beam current was used to collect images at an acceleration voltage of 5 $\mathrm{kV}$.

In vitro release of eugenol. To study the in vitro release of eugenol from emulsions, $5 \mathrm{~mL}$ of a fresh emulsion was sealed in a regenerated cellulose dialysis bag with a molecular-weightcut-off of 6000-8000 Da (Thermo Scientific Co., Marietta, OH, USA) and was dialyzed against $100 \mathrm{~mL}$ distilled water at RT with continuous stirring at a speed of $600 \mathrm{rpm}$. Aliquots of the dialysate were transferred after preset time points and diluted with ethanol for determination of eugenol using the protocol described previously. The dialysates were immediately replenished with equal volumes of distilled water to maintain the overall volume. To estimate the release of eugenol from droplets, the results were calibrated by separate dialysis experiments with the simple mixture of eugenol and water at amounts identical to the corresponding emulsion.

\section{Analysis of protein structures}

Far-ultraviolet circular dichroism spectroscopy (CD). MRP solution and fresh emulsions were diluted by 100 times using distilled water. Far-UV CD experiments were conducted using a model 202 CD spectrometer (Aviv Biomedical, Inc., Lakewood, NJ, USA) with a $0.1 \mathrm{~cm}$ quartz cell. The far-UV CD spectra were generated from averages of 3 scans collected within a wavelength range of $200-260 \mathrm{~nm}$ at a step size of $0.1 \mathrm{~nm}$ and an average time of $2 \mathrm{~s}$. Quantitative analysis of secondary structures of MRPs was enabled using the CDSSTR software available from Colorado State University (Fort Collins, CO, USA).

Hydrophobicity. To measure the overall hydrophobicity $\left(H_{\mathrm{O}}\right)$, MRP and emulsion samples with ANS as prepared in fluorescence spectroscopy experiments were diluted by 10-100 times and the fluorescence intensities were determined at excitation and emission wavelengths of 390 and $484 \mathrm{~nm}$, respectively, using the same instrument (model RF-1501, Shimadzu Corp., Tokyo, Japan). For surface hydrophobicity $\left(H_{\mathrm{S}}\right), 0.10 \mathrm{~mL}$ of an $8 \mathrm{mM}$ aqueous ANS solution was mixed with $10.0 \mathrm{~mL}$ fresh emulsions, followed by dilution and quantification of fluorescence intensity as in $H_{\mathrm{O}}$ measurements. The initial slope of the fluorescence intensity versus protein concentration $(\% \mathrm{w} / \mathrm{v})$ curve was used as an index of $H_{\mathrm{S}}{ }^{34}$

\section{Measurement of $\mathrm{O} / \mathrm{W}$ interfacial tension}

The $\mathrm{O} / \mathrm{W}$ interfacial tension $(\gamma)$ was measured with a Du Noüy ring equipped on a Cahn DCA 322 analyzer (Thermo Scientific Co., Marietta, OH, USA). $25 \mathrm{~mL}$ of eugenol and an identical volume of distilled water or the $1 \% \mathrm{w} / \mathrm{v}$ MRP stock solution at pH 8.0 were contained in a $100 \mathrm{~mL}$ beaker at RT. The platinumiridium Du Noüy ring was immersed in the bottom eugenol phase, and the force during pulling the ring from the heavier to the lighter phase at a speed of $0.8 \mathrm{~cm} \mathrm{~min}{ }^{-1}$ was monitored. The 
maximum force $\left(F_{\max }\right)$ was used to calculate $\gamma$ according to eqn (3). ${ }^{35}$

$$
\gamma=\frac{F_{\max } g}{4 \pi R}\left[0.725+\sqrt{\frac{1.452 F_{\max }}{4 \pi^{2} R^{2}\left(\rho_{1}-\rho_{2}\right)}+0.04534-\frac{1.679}{R / r}}\right]
$$

where $g$ is the acceleration due to gravity; $R$ and $r$ are the respective radius of the Du Noüy ring and the wire of the ring, which are 15.0 and $0.8 \mathrm{~mm}$, respectively, in the present study; $\rho_{1}$ and $\rho_{2}$ are the densities of the heavier and lighter phase, respectively, which are 1.06 (eugenol, supplied by the manufacturer) and 1.00 (water) or 1.01 (MRP solution) $\mathrm{g} \mathrm{cm}^{-3}$.

\section{Analyses of molecular binding}

Fluorescence spectra. $0.10 \mathrm{~mL}$ of a cationic fluorescence probe solution ( $8 \mathrm{mM}$ ANS in water) was mixed with $10.0 \mathrm{~mL}$ of the 1\% MRP stock solution. The ANS-labeled MRP solution was used to prepare emulsions as above. Fresh ANS-labeled MRP solution and emulsions were diluted by 50 times using distilled water to collect fluorescence spectra (model RF-1501, Shimadzu Corp., Tokyo, Japan). The excitation wavelength was set at $393 \mathrm{~nm}$, and the emission spectra were recorded between 400 and $650 \mathrm{~nm}$, with both the excitation and emission slit widths set at $10 \mathrm{~nm}$. The background fluorescence was calibrated using distilled water. Triple replicates were tested.

Isothermal titration calorimetry (ITC). The ITC was carried out with a Nano-ITC instrument (TA Instrument, New Castle, DE, USA). The sample cell was loaded with $500 \mu \mathrm{L}$ of a $0.5 \% \mathrm{v} / \mathrm{w}$ MRP stock solution ( $\mathrm{pH}$ 8.0) that was stirred by the injection syringe at $350 \mathrm{rpm}$ to generate rapid mixing without foaming of the protein solution. The titration by eugenol at $298 \mathrm{~K}$ was conducted for $2.5 \mu \mathrm{L}$ per injection, and the thermal effect due to mixing and dilution was calibrated by titration against a same amount of distilled water.

\section{Cytotoxicity assay}

Sample preparation. CAPE was dissolved at $20 \mathrm{mg} \mathrm{mL}^{-1}$ in eugenol or DMSO. Eugenol dissolved with CAPE was used to prepare emulsions at MRP : eugenol ratios of $1: 4,1: 10$, and $1: 20(\mathrm{~g} / \mathrm{mL})$ following the same procedures stated above. The amount of CAPE in emulsions was calculated from the total eugenol volume quantified in emulsions using a concentration of $20 \mathrm{mg} \mathrm{mL}^{-1}$, assuming the maintained proportionality after emulsification. Fresh emulsions and DMSO with CAPE were diluted with a proper amount of distilled water to obtain working solutions with an overall CAPE concentration of $0.8 \mathrm{mg}$ $\mathrm{mL}^{-1}$.

Anti-proliferation assay. Cell anti-proliferation assay followed the method of Xie et al.,$^{30}$ with slight modification. HCT116 cells were cultured in a medium supplemented with 10-time diluted FBS, 100 unit per $\mathrm{mL}$ penicillin, and $100 \mathrm{mg} \mathrm{mL}$ streptomycin under a humidified atmosphere with $5 \% \mathrm{CO}_{2}$ at $37^{\circ} \mathrm{C}$. Cells were seeded in 96-well microtiter plates at a density of 5000 cells per well in $100 \mu \mathrm{L}$ of the medium. The cells were then transferred and treated with $100 \mu \mathrm{L}$ of a sample freshly prepared from the above CAPE working solution diluted with the culture medium to 0.2 or $2 \mu \mathrm{g} \mathrm{mL} \mathrm{m}^{-1}$ of CAPE, followed by incubation for $24 \mathrm{~h}$ under the above conditions. Positive controls were the same amount of DMSO or an emulsion prepared without CAPE, while a negative control was the same volume of the culture medium. After $48 \mathrm{~h}$ incubation as above, $20 \mu \mathrm{L}$ of the CellTiter 96 Aqueous One Solution (Promega Corp., Madison, WI, USA) was added to each well and then incubated for $1 \mathrm{~h}$ at $5 \% \mathrm{CO}_{2}$ and $37{ }^{\circ} \mathrm{C}$. The absorbance at $490 \mathrm{~nm}$ was then measured with a microplate reader (Bio-Tek Instruments, Winooski, VT, USA) to estimate the normalized cell viability $\left(V_{\mathrm{N}}\right)$ using eqn (4).

$$
V_{\mathrm{N}}=\frac{A_{\mathrm{t}}-A_{\mathrm{nc}}}{A_{\mathrm{pc}}-A_{\mathrm{nc}}} \times 100 \%
$$

where $A_{\mathrm{t}}$ is the absorbance of a treatment with CAPE, $A_{\mathrm{pc}}$ is the absorbance of the positive control, and $A_{\mathrm{nc}}$ is the absorbance of the negative control. The mean and standard deviation from at least four-well replicates were calculated.

\section{Statistical analysis}

Mean and standard deviations (SD) of at least triplicates were reported. One-way analysis of variance (ANOVA) was carried out using the SPSS statistics software (IBM Corp., Armonk, NY, USA). The significance level was set at 0.05 or 0.01 .

\section{Conflicts of interest}

There are no conflicts to declare.

\section{Acknowledgements}

The authors thank the assistance of Lianger Dong in experiments. This work was supported by the National High Technology Research Development Program of China (863 Program) (No. 2013AA102204), National Natural Science Foundation of China (No. 31771898) and Special Fund for Agro-Scientific Research in the Public Interest of China (No. 201303071). T. Wang would like to thank the scholarship provided by the China Scholarship Council. Q. Zhong would like to acknowledge the support from the University of Tennessee and the USDA National Institute of Food and Agriculture Hatch Project 223984.

\section{Notes and references}

1 Q. Yang, F. Lan, Q. Yi, Y. Wu and Z. Gu, Nanoscale, 2015, 7, 17617.

2 L. Min, X. Chen, Z. Yang, X. Zhou, L. Hong and T. Ngai, ACS Appl. Mater. Interfaces, 2016, 8, 32250.

3 D. J. Mcclements, Food Emulsions: Principles, Practice and Techniques, CRC Press, 1999.

4 D. Zwicker, A. A. Hyman and F. Jülicher, Phys. Rev. E: Stat., Nonlinear, Soft Matter Phys., 2015, 92, 012317.

5 K. Pan and Q. Zhong, Annu. Rev. Food Sci. Technol., 2016, 7, 245.

6 C. W. Pouton, Adv. Drug Delivery Rev., 1997, 25, 47. 
7 C. Sabliov H. Chen and R. Yada, Nanotechnology and Functional Foods, Wiley \& Sons, 2015.

8 R. S. Makkar and J. R. Karl, Environ. Toxicol. Chem., 2003, 22, 2280.

9 G. Kortüm, W. Vogel and K. Andrussow, Pure Appl. Chem., 1960, 1, 350.

10 Y. Luo, Y. Zhang, K. Pan, F. Critzer, P. M. Davidson and Q. Zhong, J. Agric. Food Chem., 2014, 62, 4417.

11 A. Elkhattouti, N. T. Sheehan, J. Monico, H. A. Drummond, Y. Haikel, R. T. Brodell, M. Megahed and M. Hassan, Cancer Lett., 2015, 357, 83.

12 E. Filippidi, A. R. Patel, E. Bouwens, P. Voudouris and K. P. Velikov, Adv. Funct. Mater., 2014, 24, 5962.

13 T. Wang, R. Wang, Z. Chen and Q. Zhong, RSC Adv., 2016, 6, 73627.

14 S. H. Yalkowsky and Y. He, Handbook of Aqueous Solubility Data, CRC Press, 2003.

15 J. I. Park, T. D. Nguyen, J. H. Bahng, S. Srivastava, G. Zhao, K. Sun, P. Zhang, S. C. Glotzer and N. A. Kotov, Nat. Commun., 2014, 5, 3593.

16 B. P. Binks, W. G. Cho, P. D. I. Fletcher and D. N. Petsev, Langmuir, 2000, 16, 1025.

17 H. N. Po and N. M. Senozan, J. Chem. Educ., 2001, 78, 1499. 18 S. S. Wang, S. C. How, Y. D. Chen, Y. H. Tsai and J. S. Jan, J. Mater. Chem. B, 2015, 3, 5220.

19 R. Bhaskaran, M. Prabhakaran, G. Jayaraman, C. Yu and P. K. Ponnuswamy, J. Biomol. Struct. Dyn., 1996, 13, 627.

20 D. Thirumalai, G. Reddy and J. E. Straub, Acc. Chem. Res., 2011, 45, 83.
21 K. C. Powell and A. Chauhan, Langmuir, 2014, 30, 12287.

22 B. P. Binks and S. O. Lumsdon, Phys. Chem. Chem. Phys., 2000, 2, 2959.

23 H. Chen, X. Jin, Y. Li and J. Tian, RSC Adv., 2016, 6, 91060.

24 Y. Zhang, Z. Qi, D. Zheng, C. Li and Y. Liu, Biol. Trace Elem. Res., 2009, 130, 172.

25 J. R. Lakowicz, Principles of Fluorescence Spectroscopy, Plenum Press, 1983.

26 L. Nilsson and K. B. Eisenthal, Proc. Natl. Acad. Sci. U. S. A., 2005, 102, 13867.

27 G. W. Ashley, J. Henise, R. Reid and D. V. Santi, Proc. Natl. Acad. Sci. U. S. A., 2013, 110, 2318.

28 G. L. And and J. Zhou, Macromolecules, 2003, 36, 5279.

29 K. Pan, Y. Luo, Y. Gan, S. J. Baek and Q. Zhong, Soft Matter, 2014, 10, 6820.

30 M. Xie, D. Fan, Y. Chen, Z. Zhao, X. He, G. Li, A. Chen, X. Wu, J. Li and Z. Li, Biomaterials, 2016, 103, 33.

31 N. Li, N. Li, Q. Yi, K. Luo, C. Guo, D. Pan and Z. Gu, Biomaterials, 2014, 35, 9529.

32 J. Wang, Y. Wang and W. Liang, J. Controlled Release, 2012, 160, 637.

33 A. G. Gornall, C. J. Bardawill and M. M. David, J. Biol. Chem., 1949, 177, 751.

34 C. A. Haskard and L. C. Ecy, J. Agric. Food Chem., 1998, 46, 2671.

35 H. Zuidema and G. Waters, Ind. Eng. Chem., Anal. Ed., 1941, $10,2$. 